11,12

\title{
Компьютерное моделирование критического поведения двумерной слаборазбавленной антиферромагнитной модели Поттса на треугольной решетке
}

\author{
(C) А.Б. Бабаев ${ }^{1,2}$, А.К. Муртазаев ${ }^{1,3}$, Г.Я. Атаева ${ }^{1,4}$, Т.Р. Ризванова ${ }^{1}$, М.Р. Джамалудинов \\ ${ }^{1}$ Институт фризики им. Х.И. Амирханова ДагНЦ РАН, \\ Махачкала, Россия \\ ${ }^{2}$ Дагестанский государственный педагогический университет, \\ Махачкала, Россия \\ ${ }^{3}$ Дагестанский государственный университет, \\ Махачкала, Россия \\ ${ }^{4}$ Дагестанский государственный университет народного хозяйства, \\ Махачкала, Россия \\ E-mail: b_albert78@mail.ru
}

\begin{abstract}
Проведено компьютерное моделирование критического поведения двумерной слабо разбавленной антиферромагнитной модели Поттса с числом состояний спина $q=3$ на треугольной решетке. Расчеты проводились для систем с периодическими граничными условиями при концентрации спинов $p=1.0,0.9$. Рассматривались системы с линейными размерами $L \times L=N, L=9-144$. На основе теории конечноразмерного скейлинга рассчитаны статические критические индексы теплоемкости $\alpha$, восприимчивости $\gamma$, параметра порядка $\beta$ и критический индекс $v$ для радиуса корреляции.
\end{abstract}

Исследование выполнено при финансовой поддержке РФФИ в рамках научного проекта № 16-02-00214-а.

DOI: 10.21883/FTT.2018.06.45995.07M

\section{1. Введение}

Изучение тепловых и критических свойств реальных магнитных систем с присущими им примесями и дефектами структуры имеет большой теоретический и экспериментальный интерес. Связано это с тем, что неидеальные черты, являющиеся неотъемлемой частью большинства реальных твердых тел, оказывают значительное влияние на фазовые переходы (ФП) и критические явления (КЯ) в магнитных системах. В частности, присутствие немагнитных примесей в системе может изменить как род ФП, так и классы универсальности критического поведения [1-5]. Применение методов Монте-Карло (МК) позволило изучать более реалистичные модели и учитывать усложняющие факторы, всегда присутствующие в реальных материалах $[2,6]$. В качестве моделей реальных физических систем могут выступать двумерные статистические системы, такие как модель Поттса, критическое поведение которой весьма богато и интересно само по себе. Наиболее интересным на сегодня объектом для изучения является двумерная антиферромагнитная (АФ) модель Поттса на треугольной решетке. Обусловлено это тем, что для моделей Поттса треугольная решетка оказалась единственной, где наблюдается ФП при антиферромагнитном взаимодействии между ближайшими соседями. Кроме того, двумерные решеточные модели описывают большой класс реальных физических систем: слоистые магнетики, пленки, полученные путем адсорбции инертных газов на адсорбентах типа графита, сверхпроводящие пленки и др. [7].

Основной задачей данной работы являлось компьютерное моделирование критического поведения двумерной слабо разбавленной АФ модели Поттса с числом состояний спина $q=3$ на треугольной решетке, в которой вмороженный беспорядок реализован в виде немагнитных примесей.

\section{2. Двумерная АФ модель Поттса с вмороженным немагнитным беспорядком и метод исследования}

Модель Поттса представляет собой естественное обобщение модели Изинга. В модели Изинга имеется $N$ дискретных объектов, называемых узлами решетки, каждый из которых может находиться в одном из двух состояний. В модели Поттса каждый узел может находиться уже в одном из $q \geq 2$ состояний. Поэтому при построении двумерной АФ разбавленной модели Поттса с числом состояний спина $q=3$ на треугольной решетке необходимо иметь в виду следующие особенности [8].

1. В узлах треугольной решетки расположены спины $\mathrm{Si}$, которые могут находиться в одном из $q \geq 2$ состояний, и немагнитные примеси (вакансии, см. рис. 1). Немагнитные примеси распределены случайно и фиксированы на различных узлах решетки (quenched disorder).

2. Энергия парного взаимодействия принимает одно значение, если взаимодействующие узлы находятся в одинаковых состояниях (безразлично в каких именно), 


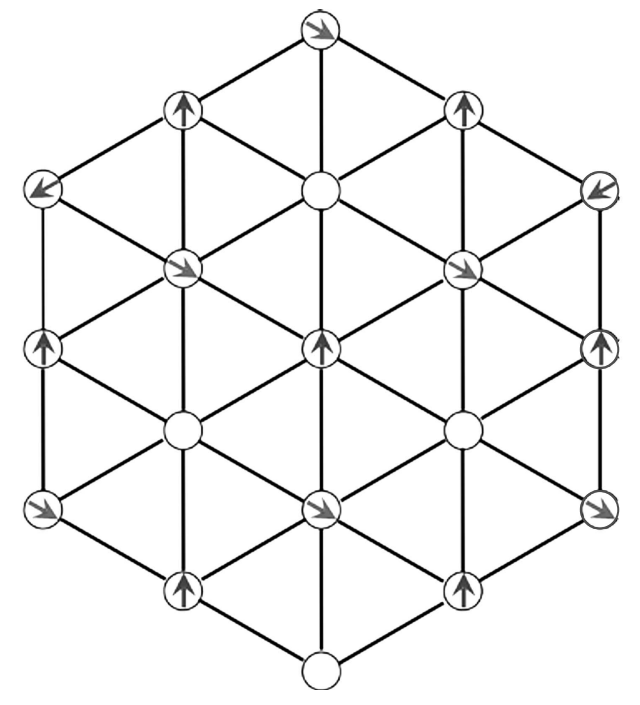

Рис. 1. Двумерная слабо разбавленная модель Поттса с числом состояний спина $q=3$ на треугольной решетке.

и другое значение, если они находятся в разных состояниях (опять же, все равно в каких именно). Энергия связи между двумя узлами равна нулю, если в одном из взаимодействующих узлов расположена немагнитная примесь.

С учетом этих особенностей микроскопический гамильтониан такой системы может быть представлен в виде

$$
H=-\frac{1}{2} J \sum_{i, j} \rho_{i} \rho_{j} \cos \theta_{i, j}, \quad S_{i}=1,2,3,
$$

где $J$ - параметр обменного АФ взаимодействия ближайших соседей $(J<0)$; $\rho_{i}=1$, если узел $i$ занят магнитным атомом, $\rho_{i}=0$, если в узле находится немагнитная примесь; $\theta_{i, j}$ - угол между взаимодействующими спинами $S_{i}-S_{j}$.

Исследование проводились на основе алгоритма Метрополиса в сочетании с кластерным алгоритмом Вольфа метода Монте-Карло. Расчеты проводились для систем с периодическими граничными условиями. Исследовались системы с линейными размерами $L \times L=N, L=9-144$. Начальные конфигурации задавались таким образом, чтобы все ближайшие соседи рассматриваемого спина находились в разных состояниях. При этом фрустрация, наблюдаемая на треугольной решетке в случае АФ-модели Изинга $(q=2)$, будет отсутствовать для модели Поттса с $q=3$ (см. рис. 1). Для вывода системы в равновесное состояние отсекался неравновесный участок длиной $\tau_{0}$ для системы с линейными размерами $L$. Этот неравновесный участок отбрасывали. Затем усреднение проводилось по участку марковской цепи длиной $\tau=200 \tau_{0}$. Для самой большой системы $L=144, \tau_{0}=2 \cdot 10^{3}$ МК шагов/спин. Кроме того, для повышения точности расчетов проводилось усреднение по 10 различным начальным конфигурациям. Затем эти данные использовались для расчета средних значений термодинамических параметров. Кроме того, для слабо разбавленных систем осуществлялось усреднение по 1000 различным конфигурациям распределения примесей в решетке.

\section{3. Результаты численного эксперимента}

Для наблюдения за температурным ходом поведения теплоемкости и восприимчивости использовались флуктуационные соотношения [9]

$$
\begin{gathered}
C=\left(N K^{2}\right)\left(\left\langle U^{2}\right\rangle-\langle U\rangle^{2}\right), \\
\chi=(N K)\left(\left\langle m_{A F}^{2}\right\rangle-\left\langle m_{A F}\right\rangle^{2}\right),
\end{gathered}
$$

где $K=|J| / k_{\mathrm{B}} T, N=p L^{2}-$ число магнитных узлов, $U$ - внутренняя энергия, $m_{A F}-$ параметр порядка системы, угловые скобки обозначают усреднение по ансамблю. В качестве параметра порядка $\left(m_{A F}\right)$ для АФ-модели Поттса использовалось следующее выражение $[10,11]$ :

$$
m_{A F}=\left\langle\frac{3}{2} \sum_{\alpha \neq \beta \neq \gamma}\left(\frac{N_{\alpha}+N_{\beta}+N_{\gamma}}{N}-\frac{1}{3}\right)\right\rangle^{1 / 2},
$$

где $N_{\alpha}=\left\{N_{1}, N_{2}, N_{3}\right\}, N_{1}$ - число спинов в состоянии с $q=1, N_{2}$ - число спинов в состоянии с $q=2, N_{3}$ число спинов в состоянии с $q=3, N_{\alpha}, N_{\beta}, N_{\gamma}$ - число спинов в подрешетках $A, B$ и $C$ соответственно.

На рис. 2 и 3 представлены характерные зависимости параметра порядка $m_{A F}$ и теплоемкости $C$ от температуры для двумерной слабо разбавленной АФ-модели Поттса для систем с линейными размерами $L=9-144$. Здесь и далее на всех рисунках погрешность данных не превышает размеров символов, используемых для

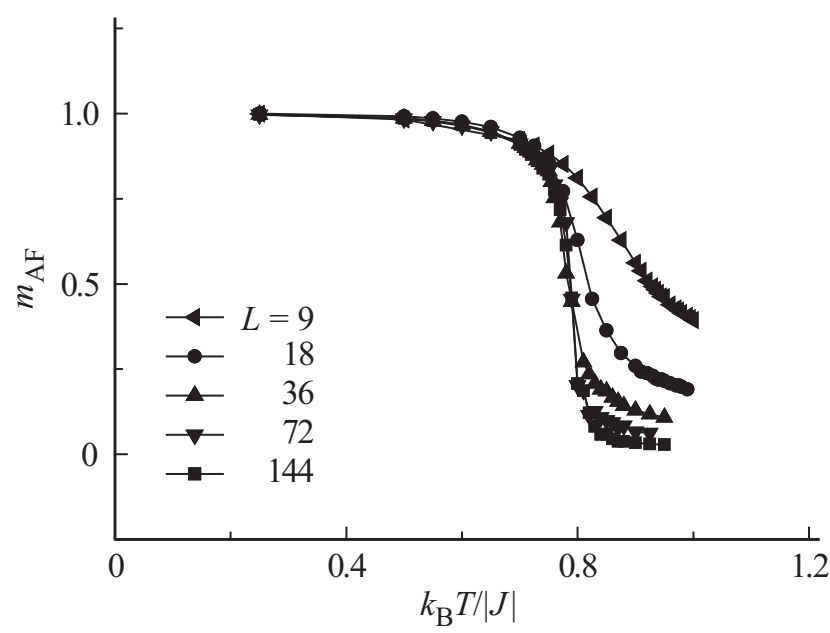

Pис. 2. Температурная зависимость параметра порядка $m_{A F}$ для двумерной слабо разбавленной АФ-модели Поттса. 


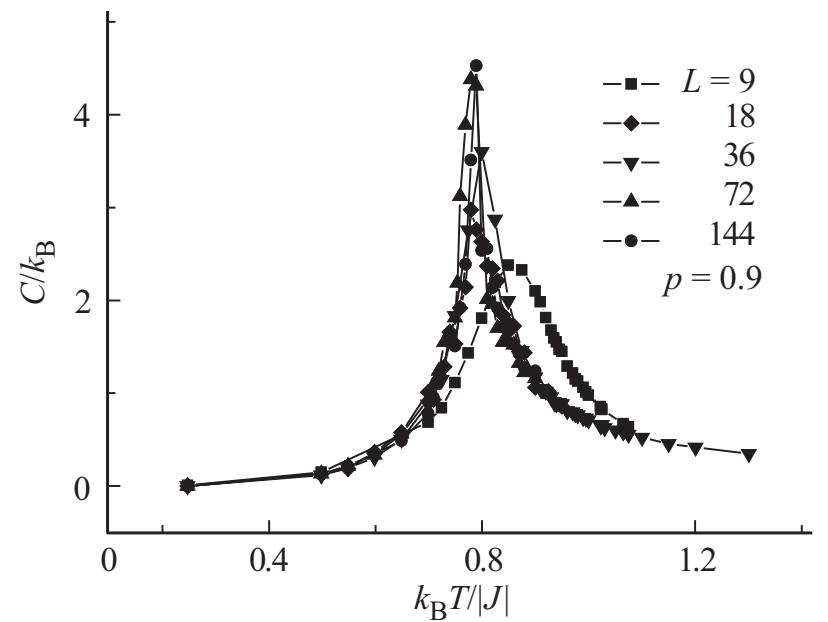

Рис. 3. Температурная зависимость теплоемкости $C$ для двумерной слабо разбавленной АФ-модели Поттса.

построения графиков. Как видно из рис. 2, для всех рассмотренных систем наблюдается поведение, характерное для ФП второго рода. Кроме того, в зависимо- стях теплоемкости $C$ от температуры для всех исследуемых нами систем проявляются четко выраженные максимумы, и эти максимумы для систем с $L \geq 9$ в пределах погрешности приходятся на одну и ту же температуру.

Для анализа характера фазового перехода и определения критических температур был использован метод кумулянтов Биндера четвертого порядка $[12,13]$. Методика определения рода ФП этим методом для магнитных систем, описываемых АФ-моделью Поттса с $q=3$ на треугольной решетке, подробно описана в работаx [14-17]. Определенные методом кумулянтов Биндера температуры фазовых переходов $T_{l}(p)$ в единицах $|J| / k_{\mathrm{B}}$ равны: $T_{l}(1.00)=0.940(1), T_{l}(0.90)=0.79(1)$.

Для всех рассмотренных систем, в которых наблюдается ФП второго рода, нами на основе теории конечноразмерного скейлинга (КРС) рассчитывались статические критические индексы (КИ) параметра порядка $\beta$, восприимчивости $\gamma$, теплоемкости $\alpha$ и КИ радиуса корреляции $v$. Из соотношений этой теории следует, что для достаточно большой системы с ПГУ при температуре $T=T_{c}$ параметр порядка $m_{A F}$, восприимчивость $\chi$ и параметр $V_{n}$ для определения критического
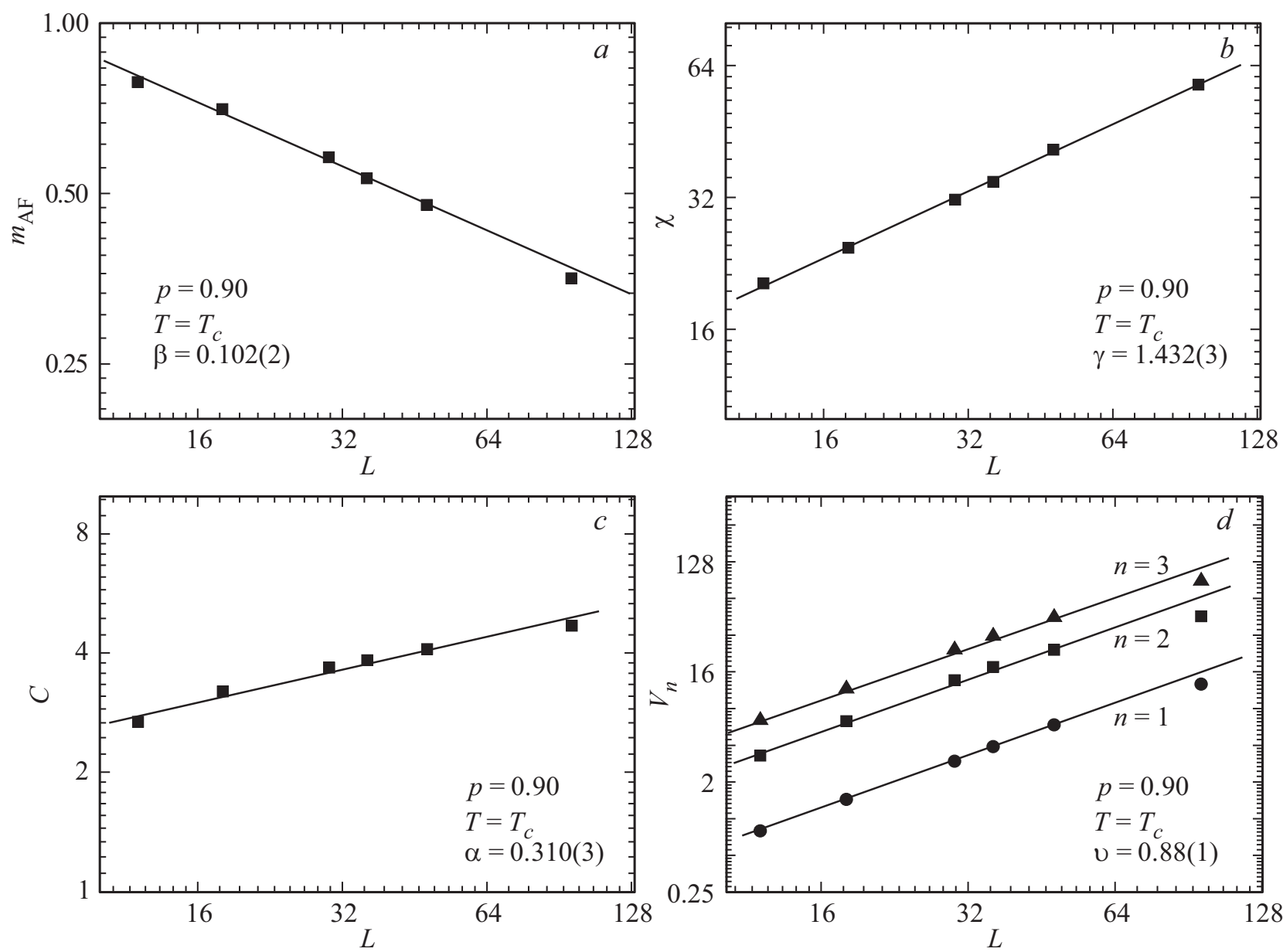

Рис. 4. Зависимость параметра порядка $m_{A F}(a)$, восприимчивости $\chi(b)$, теплоемкости $C(c)$ и параметра $V_{n}(d)$ для двумерной слабо разбавленной АФ-модели Поттса от линейных размеров системы $L$ при $T=T_{C}$. 
индекса $v$ удовлетворяют следующим аналитическим выражениям $[18,19]$

$$
\begin{aligned}
m_{A F} & \sim L^{-\beta / v}, \\
\chi & \sim L^{\gamma / \nu}, \\
V_{n} & \sim L^{1 / v},
\end{aligned}
$$

в качестве $V_{n}$ могут выступать

$$
\begin{gathered}
V_{i}=\frac{\left\langle m_{A F}^{i} E\right\rangle}{\left\langle m_{A F}^{i}\right\rangle}-\langle E\rangle, \quad(i=1,2), \\
V_{3}=\frac{d U_{L}}{d \beta} \\
=\frac{1}{3\left\langle m_{A F}^{3}\right\rangle^{2}}\left[\left\langle m_{A F}^{4}\right\rangle\langle E\rangle-2 \frac{\left\langle m_{A F}^{4}\right\rangle\left\langle m_{A F}^{4} E\right\rangle}{\left\langle m_{A F}^{2}\right\rangle^{2}}+\left\langle m_{A F}^{4} E\right\rangle\right],
\end{gathered}
$$

где $\beta=1 / T, T-$ температура.

Для аппроксимации температурной зависимости теплоемкости от $L$ как правило используются другие выражения, например [9]

$$
C_{\max }(L)=C_{\max }(L=\infty)-A L^{\alpha / v},
$$

где $A$ - некоторый коэффициент.

Для расчета КИ $\beta, \gamma, \alpha$ и $v$ строились зависимости $m_{A F}, \chi, C$ и $V_{n}$ от $L$. На рис. $4, a-d$ в двойном логарифмическом масштабе представлены характерные зависимости параметра порядка $m_{A F}$, восприимчивости $\chi$, теплоемкости $C$ и параметра $V_{n}$ для определения критического индекса радиуса корреляции, от линейных размеров решетки $L$ для двумерной АФ слабо разбавленной модели Поттса на треугольной решетке при $T=T_{C}$ и $p=0.9$. Обратим внимание на то, что данные, полученные для всех рассмотренных термодинамических параметров, не значительно отклоняются от прямой при малых значениях $L$. Очевидно, что использованное нами для усреднения количество различных начальных конфигураций и размеры $L>9$ изучаемых систем позволяют достичь асимптотического критического режима. Очень важным моментом является и то, что индекс $v$ вычислялся непосредственно из результатов численного эксперимента в рамках данного исследования, тогда как во многих других работах этот индекс обычно определяется из различных скейлинговых соотношений.

Анализ данных, выполненный с использованием нелинейного метода наименьших квадратов, позволил определить значения $\frac{\alpha}{v}=0.352(3), \frac{\beta}{v}=0.116(2)$, $\frac{\gamma}{v}=1.627(3)$ и $\frac{1}{v}=1.136(3)$. Затем, с использованием значений $v=0.832(2)$, полученных в рамках данного исследования, были получены следующие индексы: $\alpha=0.310(3), \beta=0.102(2), \gamma=1.432(3), \quad v=0.88(1)$. Полученные критические индексы в пределах погрешности удовлетворяют соотношениям теории КРС.

\section{4. Заключение}

Таким образом, в настоящей работе с соблюдением единой методики проведено компьютерное моделирование критического поведения двумерной слабо разбавленной АФ-модели Поттса с $q=3$ на треугольной решетке при концентрации спинов $p=0.90$. На основе теории конечно-размерного скейлинга рассчитан весь набор критических индексов. Значения критических индексов $\alpha, \beta, \gamma$ и $\nu$, полученные в рамках данного исследования, показывают, что класс универсальности данной модели при слабом разбавлении характеризуется новым набором критических индексов.

\section{Список литературы}

[1] В.С. Доценко. УФН 165, 5, 481 (1995).

[2] Р. Фольк, Ю. Головач, Т. Яворский. Успехи физических наук 173, 175 (2003).

[3] А.К. Муртазаев, А.Б. Бабаев. Письма в ЖЭТФ 99, 9, 618 (2014) [JETP Lett. 99, 535 (2014).]

[4] А.К. Муртазаев, А.Б. Бабаев. ЖЭТФ 143, 1, 116 (2013) [J. Experim. Theor. Phys. 116, 101 (2013).]

[5] П.В. Прудников, М.А. Медведева. Физика низких температур 40, 5, 570 (2014).

[6] A.K. Murtazaev, A.B. Babaev, G.Y. Aznaurova. Solid State Phenomena 168-169, 357 (2011).

[7] D.P. Landau, K. Binder. A Cuide to Monte-Carlo Simulations in Statistical Physics, Cambridge University Press, N.Y. (2009).

[8] F.Y. Wu. Rev. Mod. Phys. 54, 235 (1982).

[9] P. Peczac, A.M. Ferrenberg, D.P. Landau. Phys. Rev. B 43, 6087 (1991).

[10] Y. Saito. J. Phys. A 15, 1885 (1982).

[11] А.Б. Бабаев, А.К. Муртазаев, Э.М. Сулейманов, Т.Р. Ризванова, ФТТ 58, 10, 2001 (2016).

[12] K. Eichhorn, K. Binder. J. Phys.: Condens. Matter 8, 5209 (1996).

[13] D. Loison, K.D. Schotte. Eur. Phys. J. B 5, 735 (1998).

[14] A.K. Murtazaev, A.B. Babaev. J. Experim. Theor. Phys. 115, 6, $1042(2012)]$.

[15] А.К. Муртазаев, А.Б. Бабаев, М.А. Магомедов, Ф.А. Кассан-Оглы, А.И. Прошкин. Письма в ЖЭТФ 100, 4, 267 (2014) [JETP Lett. 100, 242 (2014)].

[16] А.К. Муртазаев, А.Б. Бабаев. Изв. РАН. Сер. физ. 77, 10, 1476 (2013).

[17] А.К. Муртазаев, А.Б. Бабаев, Г.Я. Атаева. Физика низких температур 39, 2, 194 (2013).

[18] D. Loison. Phys. Lett. A 257, 83 (1999).

[19] M.E. Fisher, M.N. Barber. Phys. Rev. Lett. 28, 1516 (1972). 